\title{
Development and Standardization of a Simple and Quick Screening Protocol for Arsenic Phyto-toxicity Tolerance at Seedling Stage in Rice
}

\author{
Md. Abu Syed ${ }^{1, *}$, K. M. Iftekharuddaula ${ }^{1}$, Md. Golam Rasul' ${ }^{2}$, G. K. M. Mustafizur Rahmam ${ }^{3}$, \\ Golam M. Panaullah ${ }^{4}$, John M. Duxbury ${ }^{5}$, Mohammod Hossain ${ }^{6}$, Partha S. Biswas ${ }^{1}$ \\ ${ }^{1}$ Plant Breeding Division, Bangladesh Rice Research Institute (BRRI), Bangladesh \\ ${ }^{2}$ Department of Genetics and Plant Breeding, Bangabandhu Sheikh Mujibur Rahman Agricultural University (BSMRAU), Bangladesh \\ ${ }^{3}$ Department of Soil Science, Bangabandhu Sheikh Mujibur Rahman Agricultural University (BSMRAU), Bangladesh \\ ${ }^{4}$ Former Director Research, Bangladesh Rice Research Institute (BRRI), Bangladesh \\ ${ }^{5}$ Department of Crop and Soil Sciences, Cornell University, USA \\ ${ }^{6}$ Plant Pathology Division, Bangladesh Rice Research Institute (BRRI), Bangladesh
}

Copyright $\mathrm{C} 2019$ by authors, all rights reserved. Authors agree that this article remains permanently open access under the terms of the Creative Commons Attribution License 4.0 International License

\begin{abstract}
Cultivation with Arsenic (As) contaminated water severely affects growth of rice plants that results into reduced grain yield. This study was carried out to develop a rapid and effective method of screening rice varieties and/or segregating population against arsenic phyto-toxicity tolerance. Sodium arsenate was used as arsenic source. Ten pre-germinated seeds of each variety were grown on styro-foam placed on a tray filled with either phosphate-free nutrient solution (control) or the same nutrient solution supplemented with di-sodium hydrogen arsenate (treatment). The trays were kept at controlled environmental condition in a growth chamber at $26 \pm 1{ }^{\circ} \mathrm{C}$ with $70 \%$ humidity and 3500 lux. After 7 days of growth, standard growth parameters such as shoot length, root length, root-shoot biomass were recorded and percentages reduction for shoot length, root length and root shoot biomass compared to those of control were calculated. Arsenic stress reduced the phenotypic expression of all three seedling traits but the effect of arsenic was more prominent on root length compared to the shoot length and root-shoot biomass. The dose response experiment showed that the $0.75-1.25 \mathrm{mgL}^{-1}$ Arsenic concentrations had much promise to discriminate rice genotypes for arsenic phyto-toxicity tolerance at early growth stage. The validation experiment with the $0.75-1.25 \mathrm{mgL}^{-1}$ Arsenic doses on a set of 20 genotypes identified $1.25 \mathrm{mgL}^{-1}$ Arsenic could effectively and efficiently differentiate rice genotypes, particularly for tolerance in shoot length and root-shoot biomass reduction, where $1.0 \mathrm{mgL}^{-1}$ Arsenic was found sufficient for discriminating varieties for tolerance in root length reduction. The rice variety BRRI dhan 47 showed more
\end{abstract}

tolerance against Arsenic contaminated water.

Keywords Arsenic, Hydroponic, Reactive Oxygen Species, Least significant Difference, Arsenic Phyto-toxicity, Rice

\section{Introduction}

Arsenic (As), a non-essential element for plant growth, it is generally phyto-toxic and is expected to negatively affect plant growth [1-3]. As a result of many negative effects, arsenic causes reduced growth of plants. The primary response of plants is the generation of reactive oxygen species (ROS) after exposure to arsenic. ROS can directly damage proteins, amino acids and nucleic acids and cause peroxidation of membrane lipids [4]. Arsenic accumulates in the plant tissue and stimulates peroxidase synthesis during the early phases of plant development, long before the appearance of visible changes [5-7]. Rice yield and biomass production are greatly reduced at elevated Arsenic concentrations, often leading to death of plant population [8-10]. Long-term irrigated soils subjected to Arsenic contaminated ground water may create a significant risk to animal and human health via soil-crop transfer of Arsenic. In addition, consumption of excess Arsenic from groundwater water has caused human health problems in several countries [11-14].Therefore, many researchers in the entire world have shown keen interest in arsenic toxicity, screening and selecting arsenic tolerant and remediative plants and mechanisms related to 
their detoxification.

Since there are good evidences that metal tolerance is under genetic control [17]. To facilitate breeding programmes, a rapid, non-destructive, inexpensive and repeatable seedling-based bioassay is required for selection of tolerant genotypes from early segregating generations [18]. Field screening methodology is less precise, less efficient and, therefore, less attractive to breeders since this method is challenged by many factors, including soil heterogeneity in physical and chemical characteristics, tedious excavations of roots, low screening rate, and time-consuming when a large number of genotypes are under evaluation. Therefore, a rapid and effective screening system is needed to discriminate tolerant and sensitive genotypes. Hydroponics, on the other hand, is more attractive than the soil-based screening because it allows rapid evaluation of a large number of genotypes in a shorter time on the basis of relative growth rate and toxicity [19]. Besides, accurate phenotypic data are expected from hydroponics because of the tight control of the screening conditions. Importantly, hydroponics enables observations to be made of intra- and inter-specific genetic variation in plant responses, in terms of levels of tolerance shown and the specific tolerance mechanisms that are employed. The use of hydroponic culture has been suggested as a means of assessing the plant tolerance to the toxic elements or the efficiency in mineral utilization [20-22]. Hydroponic techniques have been utilized by many scientists to identify and understand the tolerance mechanism under excess heavy metals in plants. Meharg [23] reported hydroponic culture as a suitable way to screen and identify heavy metal tolerant and remediative plants before field studies.

Improving arsenic tolerance in rice plants is the most suitable way to decrease destructive effects of arsenic toxicity [24-25]. Since tolerance is genetically controlled, screening and selection of Arsenic tolerant rice genotypes at seedling stage under hydroponics condition can lead to the development of tolerant cultivars/varieties against excess arsenic because hydroponics provides easy access to root system, exact control over available nutrients and $\mathrm{pH}$ and non-destructive measurements of tolerance. But, rapid screening procedure for arsenic phyto-toxicity tolerance in rice is available only for root length parameter [20]. But only root length parameter is not sufficient for arsenic tolerant variety development. Three growth parameters (root length, shoot length and root-shoot biomass) were considered in this study. Considering the above fact the present study has been undertaken with the following objectives: i) to develop and standardize a quick and short-cut screening protocol; ii) to discriminate rice varieties and/or segregating population into tolerant and susceptible groups effectively and efficiently with a single dose of arsenic treatment under controlled condition.

\section{Materials and Methods}

The experiment was conducted at the screening house of Plant breeding field Laboratory, Bangladesh Rice Research Institute (BRRI), Gazipur-1701.

\subsection{Plant Materials}

Two arsenic tolerant (BRRI dhan47 and BRRI dhan54) and two sensitive rice varieties (BRRI dhan29 and BRRI dhan45) identified from previous study [26] were used in dose response experiment. Another twenty arsenic tolerant and sensitive rice varieties including some IRRI lines, US and Chinese cultivars were used for validation of identified in the dose response experiment. The seeds were collected from the Plant Breeding Division and Gene Bank of Bangladesh Rice Research Institute (BRRI), Gazipur-1701.

\subsection{Experimental Design and Treatments}

Dose response experiment The experiment was laid out in a Randomized Complete Block Design (RCBD).The treatments were arranged in a $4 \times 7$ factorial scheme comprising four rice genotypes (BRRI dhan29, BRRI dhan45, BRRI dhan47 and BRRI dhan54) and seven concentrations of arsenic in solution $(0,0.25,0.50,0.75$, $1.0,1.25$ and $1.50 \mathrm{mg} \mathrm{L}^{-1}$ Arsenic) with four replications. Layout of dose response experiment was as below: 
Dose validation experiment The experiment for dose validation with 20 genotypes was conducted for 3 levels of Arsenic $\left(0.75,1.0\right.$ and $\left.1.25 \mathrm{mg} \mathrm{L}^{-1}\right)$ in three replications. Arsenic (As) was used in the form of $\mathrm{NaH}_{2} \mathrm{AsO}_{4}($ Analytical reagent grade; $\mathrm{BDH}$ ). The phosphate-free nutrient solution consisted of $0.1 \mathrm{mM} \mathrm{Mg}^{2+}$ and $\mathrm{SO}^{2-}, 0.2 \mathrm{mM} \mathrm{Ca}^{2+}$ and K${ }^{+}$and $0.6 \mathrm{mM} \mathrm{NO}_{3}^{-}[27]$ was used to grow seedlings. Layout of dose validation experiment was given below:

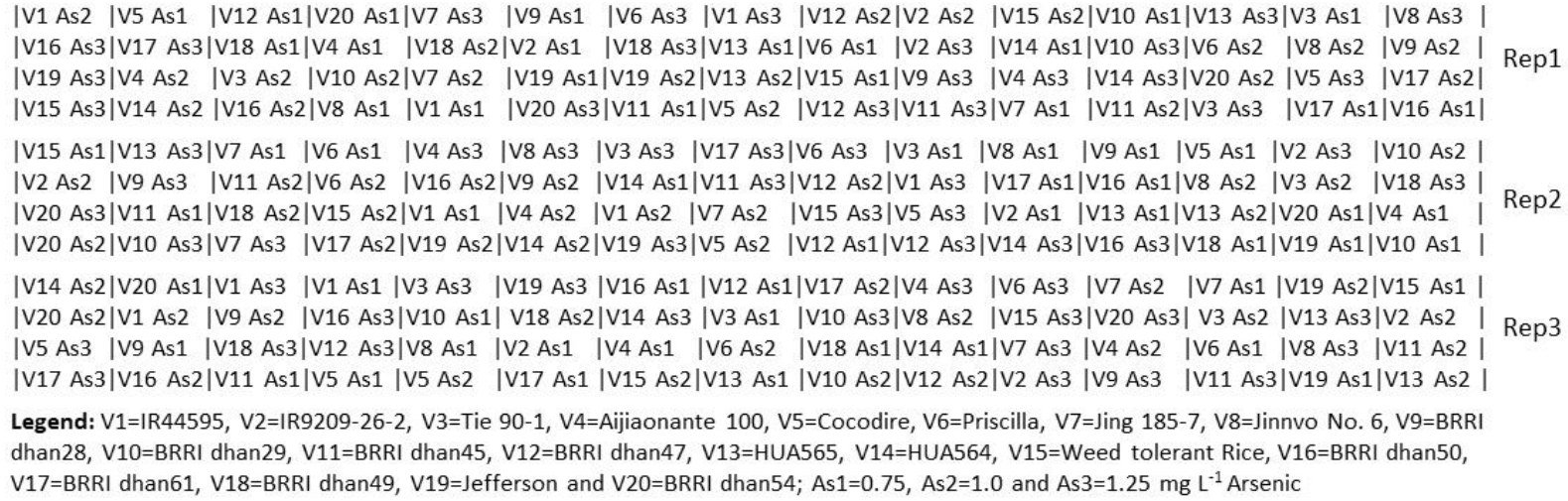

\subsection{Experimental Set-Up}

Seeds were disinfected with Vitavex for 2 hours and soaked overnight in petridishes after washing with distilled water and then incubated at $30^{\circ} \mathrm{C}$ for 48 hours. Ten pre-germinated seeds were placed on styrofoam floats with a mosquito net placed at the bottom. Then the floats with pre-germinated seeds were placed on the tray filled with either phosphate-free nutrient solution (control) or the same nutrient solution supplemented with di-sodium hydrogen arsenate (treatment). The trays were kept in a growth chamber at $26 \pm 1{ }^{\circ} \mathrm{C}$ with $70 \%$ humidity and $3500 \mathrm{lux}$, white fluorescent light $\left(55 \mu \mathrm{mol} \mathrm{m} \mathrm{s}^{-2}\right)$. After 7 days of growth, shoot length $(\mathrm{cm})$ wasmeasured from the base of the culm to the tip of the longest leaf and root length $(\mathrm{cm})$ was recorded from the root-shoot junction to the tip of the longest root. Root and shoot samples were dried in an oven at $50^{\circ} \mathrm{C}$ for 96 hours and then weighed to determine their biomass (mg).

\subsection{Statistical Analysis}

The rate of reduction in shoot length, root length and root-shoot biomass was estimated as the percentage of reduction in shoot length, root length and root shoot biomass, respectively compared to control following a formula given as,

Rate of reduction $(\%)=1-($ Growth with arsenic - Growth without arsenic)*100

The data were subjected to analyses of variance (ANOVA) to determine the significance of individual effects (genotypes and arsenic concentration) and genotype $\mathrm{x}$ Arsenic treatment interactions. Least significant differences (LSD) were calculated at $\mathrm{P}=0.05$ for comparisons between mean values. The ANOVA and LSD test were performed using Statistix 10 software (Tallahassee, FL, USA) and rate of reduction, standard deviation (SD), range, coefficient of variation (CV) were calculated using Excel 2007.

\section{Results}

The analysis of variance (ANOVA) of arsenic dose response and dose validation experiments revealed significant variation in shoot length reduction, root length reduction and root-shoot reduction among various genotypes and different arsenic concentrations. The interaction effects were also significant (Table 1).

Table 1. Mean squares of the analysis of variance for shoot length reduction (\%), root length reduction (\%), root-shoot biomass reduction (\%) in dose response experiment

\begin{tabular}{|c|c|c|c|c|}
\hline \multirow{2}{*}{ Sources } & \multirow{2}{*}{ Degree of freedom } & \multicolumn{3}{|c|}{ Reduction (\%) } \\
\hline & & Shoot length & Root length & Root-shoot biomass \\
\hline Genotypes (G) & 3 & $887.51^{* * *}$ & $3949.27 * * *$ & $250.98 * * *$ \\
\hline Concentrations (C) & 5 & $3740.06^{* * *}$ & $1997.00 * * *$ & $2470.68 * * *$ \\
\hline $\mathrm{G} \times \mathrm{C}$ & 15 & $17.57 * * *$ & $35.57 * * *$ & $36.02 \mathrm{NS}$ \\
\hline Error & 69 & 5.15 & 5.02 & 21.61 \\
\hline Coefficient of variation $(\%)$ & & 27.24 & 21.45 & 36.12 \\
\hline Heritability (h2b) & & 97.72 & 99.49 & 72.63 \\
\hline
\end{tabular}

${ }^{* * *}$ Significant at $0.1 \%$ level of significance, ${ }^{\mathrm{NS}}$ Non-significant 


\subsection{Shoot Length Reduction}

Genotypic mean comparison showed that the lowest shoot length reduction (47.59\%) was in BRRI dhan47 which was statistically different from other genotypes. The maximum shoot length reduction was observed in the genotype BRRI dhan54 (61.74\%), followed by BRRI dhan29 (58.19\%), and BRRI dhan45 (57.66\%) (Table 2). Concentration means revealed that shoot length reduction was increased under increasing of Arsenic concentrations and was also significantly differed from each other. It was maximum (71.69\%) at $1.50 \mathrm{mgL}^{-1}$ followed by $1.25 \mathrm{mgL}^{-1}$
(64.54\%).Shoot length reduction was very close and statistically similar at $1.00 \mathrm{mgL}^{-1}(62.18 \%)$ to that at 0.75 $\mathrm{mgL}^{-1}(61.43 \%)$ level of applied arsenic. The lower reduction was found at $0.25 \mathrm{mgL}^{-1}$ (28.91\%) followed by $0.50 \mathrm{mgL}^{-1}(49.01 \%)$. The shoot length reduction among genotypes except BRRI dhan 47 was almost close to each other at $0.25 \mathrm{mg} \mathrm{L}^{-1}, 0.50 \mathrm{mg} \mathrm{L}^{-1}$ and $1.50 \mathrm{mg} \mathrm{L}^{-1}$ levels of arsenic. But at the concentration from $0.75 \mathrm{mg} \mathrm{L}^{-1}$ to 1.25 $\mathrm{mg} \mathrm{L}^{-1}$ of Arsenic, the genotypic difference was apparent and significant. The range along with standard deviation was also the highest at the Arsenic concentration of 0.75 $\mathrm{mg} \mathrm{L}^{-1}$ to $1.25 \mathrm{mg} \mathrm{L}^{-1}$.

Table 2. Mean of shoot length reduction (\%) over different concentration $\left(\mathrm{mg} \mathrm{L}^{-1}\right)$ of arsenic in dose response experiment

\begin{tabular}{|c|c|c|c|c|c|c|c|}
\hline \multirow{2}{*}{ Genotypes } & \multicolumn{6}{|c|}{ Arsenic concentration $\left(\mathrm{mg} \mathrm{L}^{-1}\right)$} & \multirow{2}{*}{ Genotypic means } \\
\hline & 0.25 & 0.50 & 0.75 & 1.0 & 1.25 & 1.50 & \\
\hline BRRI dhan29 & $27.9 \mathrm{bc}$ & $49.6 \mathrm{a}$ & $64.7 \mathrm{ab}$ & $65.3 \mathrm{~b}$ & $66.7 \mathrm{~b}$ & $74.8 \mathrm{a}$ & 58.2 \\
\hline BRRI dhan45 & $29.4 \mathrm{ab}$ & $52.2 \mathrm{a}$ & $61.8 \mathrm{~b}$ & $62.4 \mathrm{~b}$ & $66.6 \mathrm{~b}$ & $73.6 \mathrm{a}$ & 57.7 \\
\hline BRRI dhan47 & $24.5 \mathrm{c}$ & $41.5 \mathrm{~b}$ & $50.3 \mathrm{c}$ & $51.6 \mathrm{c}$ & $54.8 \mathrm{c}$ & $62.9 \mathrm{~b}$ & 47.6 \\
\hline BRRI dhan54 & $33.7 \mathrm{a}$ & $52.8 \mathrm{a}$ & $68.9 \mathrm{a}$ & $69.5 \mathrm{a}$ & $70.0 \mathrm{a}$ & $75.5 \mathrm{a}$ & 61.8 \\
\hline Concentration means & 28.91 & 49.01 & 61.43 & 62.18 & 64.54 & 71.69 & \\
\hline $\operatorname{LSD}(0.05)$ & \multicolumn{6}{|c|}{1.60} & 1.31 \\
\hline Standard Deviation & 3.82 & 5.22 & 7.97 & 7.66 & 6.69 & 5.92 & \\
\hline Range & $24.5-33.7$ & $41.5-52.8$ & $50.3-68.9$ & $51.6-69.5$ & $54.8-70.0$ & $62.9-75.4$ & \\
\hline
\end{tabular}

Within column different letters indicate significance differences among rice varieties (LSD test: $\mathrm{P}<0.05$ )

Table 3. Mean shoot length reduction (\%) over three levels of arsenic concentrations ( $\left.\mathrm{mg} \mathrm{L}^{-1}\right)$ of 20 rice genotypes

\begin{tabular}{|c|c|c|c|c|}
\hline \multirow{2}{*}{ Genotypes } & \multicolumn{3}{|c|}{ Arsenic concentration $\left(\mathrm{mg} \mathrm{L}^{-1}\right)$} & \multirow{2}{*}{ Genotypic Mear } \\
\hline & 0.75 & 1.0 & 1.25 & \\
\hline IR44595 & $46.06 \mathrm{ij}$ & $51.55 \mathrm{kl}$ & $56.94 \mathrm{ghi}$ & 51.51 \\
\hline IR9209-26-2 & $59.54 \mathrm{~b}$ & $65.69 \mathrm{ab}$ & $68.26 \mathrm{~b}$ & 64.51 \\
\hline Tie 90-1 & $56.07 \mathrm{bc}$ & $60.29 \mathrm{~d}$ & $64.20 \mathrm{c}$ & 60.18 \\
\hline Aijiaonante 100 & 49.15 ghi & 57.20 efg & 59.76 ef & 55.39 \\
\hline Cocodire & $64.61 \mathrm{a}$ & $55.97 \mathrm{fgh}$ & $61.45 \mathrm{de}$ & 57.33 \\
\hline Priscilla & 53.24 cdefg & $54.43 \mathrm{hij}$ & $56.77 \mathrm{ghi}$ & 54.81 \\
\hline Jing 185-7 & $55.71 \mathrm{bc}$ & 57.34 ef & $61.98 \mathrm{~d}$ & 58.34 \\
\hline Jinnvo No. 6 & $44.89 \mathrm{j}$ & $48.58 \mathrm{~m}$ & $52.44 \mathrm{j}$ & 48.67 \\
\hline BRRI dhan28 & 50.17 fghi & $52.70 \mathrm{jk}$ & $59.94 \mathrm{ef}$ & 54.26 \\
\hline BRRI dhan29 & $59.67 \mathrm{~b}$ & $63.98 \mathrm{bc}$ & $67.70 \mathrm{~b}$ & 63.68 \\
\hline BRRI dhan 45 & $52.05 \mathrm{cdefg}$ & $55.89 \mathrm{fgh}$ & $58.41 \mathrm{fg}$ & 55.46 \\
\hline BRRI dhan 47 & $39.03 \mathrm{k}$ & $44.87 \mathrm{n}$ & $46.86 \mathrm{k}$ & 43.58 \\
\hline HUA565 & $54.87 \mathrm{~cd}$ & 55.33 ghi & $56.14 \mathrm{hi}$ & 55.43 \\
\hline HUA564 & 51.52 defgh & $53.59 \mathrm{ij}$ & $58.41 \mathrm{fg}$ & 54.53 \\
\hline Weed tolerant Rice & 51.54 defgh & $56.47 \mathrm{fg}$ & $57.57 \mathrm{gh}$ & 55.22 \\
\hline BRRI dhan50 & $47.52 \mathrm{hij}$ & $50.221 \mathrm{~m}$ & $55.51 \mathrm{i}$ & 51.07 \\
\hline BRRI dhan61 & $53.71 \mathrm{cdef}$ & $66.73 \mathrm{a}$ & $73.93 \mathrm{a}$ & 64.76 \\
\hline BRRI dhan 49 & $54.06 \mathrm{cdef}$ & - & - & 54.07 \\
\hline Jefferson & 50.49 efgh & $59.12 \mathrm{de}$ & $62.09 \mathrm{~d}$ & 57.20 \\
\hline BRRI dhan54 & - & $56.04 \mathrm{fgh}$ & $62.73 \mathrm{~cd}$ & 59.41 \\
\hline Concentration means & 52.41 & 56.42 & 60.27 & \\
\hline LSD value at 0.05 & & 0.66 & & 1.63 \\
\hline Standard deviation & 5.68 & 5.59 & 5.93 & \\
\hline Range & $39.03-64.61$ & $44.87-66.73$ & $46.86-73.93$ & \\
\hline
\end{tabular}

Within column different letters indicate significance differences among rice varieties (LSD test: $\mathrm{P}<0.05$ ) 
Validation trial with $0.75 \mathrm{mg} \mathrm{L}^{-1}, 1.0 \mathrm{mg} \mathrm{L}^{-1}$ and $1.25 \mathrm{mg}$ $\mathrm{L}^{-1}$ Arsenic over a set of 20 diverse genotypes showed that the minimum mean shoot length reduction was with BRRI dhan47 (43.58\%) followed by Jinnvo no. 6 (48.67\%), while the maximum mean reduction was found with BRRI dhan61 (64.76\%) followed by IR9209-26-2 (64.51\%) and BRRI dhan29 (63.68\%) (Table 3). The shoot length reduction was increased with increasing arsenic concentration. The highest mean reduction $(60.27 \%)$, the highest standard deviation (5.93) and the largest range (46.86-73.93) in shoot length reduction were observed at $1.25 \mathrm{mg} \mathrm{L}^{-1}$ Arsenic among all the Arsenic concentrations. The highest difference in shoot length reduction between the known tolerant variety (BRRI dhan47) and sensitive varieties (BRRI dhan29 and BRRI dhan54) was also found at $1.25 \mathrm{mg} \mathrm{L}^{-1}$ level of Arsenic compared to other concentrations.

\subsection{Root Length Reduction}

Genotypic mean of root length reduction was found least in BRRI dhan47 (53.57\%), which was statistically different from other genotypes in dose response experiment. Rice varieties BRRI dhan $45(80.35 \%)$ and BRRI dhan29 (79.44\%) showed the highest root length reduction that was statistically similar followed by BRRI dhan54 (77.75\% )
(Table 4). Concentration means showed that root length reduction was the minimum at $0.25 \mathrm{mg} \mathrm{L}^{-1}$ and the maximum at $1.50 \mathrm{mg} \mathrm{L}^{-1}$ although all concentration means were statistically different from each other. The maximum statistical differentiation among genotypes was found at $1.25 \mathrm{mg} \mathrm{L}^{-1}$ followed by $1.50 \mathrm{mg} \mathrm{L}^{-1}, 1.0 \mathrm{mg} \mathrm{L}^{-1}$ and 0.25 $\mathrm{mg} \mathrm{L}^{-1}$. The maximum differentiation at $0.25 \mathrm{mg} \mathrm{L}^{-1}$ and $1.50 \mathrm{mg} \mathrm{L}^{-1}$ doses were actually varietal difference probably due to lower and higher dose. The highest range and standard deviation were observed at the Arsenic concentration of $0.75 \mathrm{mg} \mathrm{L}^{-1}$ followed by $0.5 \mathrm{mg} \mathrm{L}^{-1}$ and $1.25 \mathrm{mg} \mathrm{L}^{-1}$.

In validation trial, BRRI dhan 47 exhibited significantly least root length reduction (58.89\%) among 20 genotypes (Table 5). Among three levels of arsenic, the highest mean root length reduction $\left(78.75 \%\right.$ ) was found at $1.25 \mathrm{mg} \mathrm{L}^{-1}$ Arsenic. The differences in percent of root length reduction between BRRI dhan47 and BRRI dhan45, and BRRI dhan 47 and BRRI dhan 54 were the maximum at $1.00 \mathrm{mg}$ $\mathrm{L}^{-1}$ Arsenic but between BRRI dhan 47 and BRRI dhan29, it was at $0.75 \mathrm{mg} \mathrm{L}^{-1}$. The highest standard deviation (7.83) between the genotypes was found at $0.75 \mathrm{mg} \mathrm{L}^{-1}$ Arsenic, although $1.00 \mathrm{mg} \mathrm{L}^{-1}$ Arsenic had the reduction rate ranging from (59.77\%-87.56\%), which was almost similar to that of $1.25 \mathrm{mg} \mathrm{L}^{-1}$ Arsenic.

Table 4. Mean of root length reduction (\%) over different concentration $\left(\mathrm{mg} \mathrm{L}^{-1}\right)$ of arsenic in dose response experiment

\begin{tabular}{|c|c|c|c|c|c|c|c|}
\hline \multirow{2}{*}{ Genotypes } & \multicolumn{6}{|c|}{ Arsenic concentration $\left(\mathrm{mg} \mathrm{L}^{-1}\right)$} & \multirow{2}{*}{ Genotypic means } \\
\hline & 0.25 & 0.50 & 0.75 & 1.0 & 1.25 & 1.50 & \\
\hline BRRI dhan29 & $54.4 \mathrm{~b}$ & $78.8 \mathrm{a}$ & $80.0 \mathrm{a}$ & $85.3 \mathrm{a}$ & $87.1 \mathrm{~b}$ & $91.0 \mathrm{a}$ & 79.4 \\
\hline BRRI dhan45 & $61.7 \mathrm{a}$ & $75.8 \mathrm{a}$ & $79.6 \mathrm{a}$ & $84.8 \mathrm{a}$ & $89.6 \mathrm{a}$ & $90.6 \mathrm{ab}$ & 80.3 \\
\hline BRRI dhan 47 & $37.8 \mathrm{c}$ & $47.3 \mathrm{~b}$ & $48.7 \mathrm{~b}$ & $57.1 \mathrm{c}$ & $58.7 \mathrm{~d}$ & $71.8 \mathrm{c}$ & 53.6 \\
\hline BRRI dhan54 & $59.9 \mathrm{a}$ & $74.3 \mathrm{a}$ & $77.6 \mathrm{a}$ & $81.4 \mathrm{~b}$ & $83.7 \mathrm{c}$ & $89.5 \mathrm{~b}$ & 77.8 \\
\hline Concentration means & 53.47 & 69.06 & 71.50 & 77.15 & 79.79 & 85.71 & \\
\hline $\operatorname{LSD}(0.05)$ & \multicolumn{6}{|c|}{1.58} & 1.29 \\
\hline Standard Deviation & 10.92 & 14.63 & 15.22 & 13.46 & 14.27 & 9.31 & \\
\hline Range & $37.8-61.7$ & $47.3-78.8$ & $48.7-80.0$ & $57.1-85.3$ & $58.7-89.6$ & $71.8-91.0$ & \\
\hline
\end{tabular}

Within column different letters indicate significance differences among rice varieties (LSD test: $\mathrm{P}<0.05$ ) 
Table 5. Mean root length reduction (\%) over three level of arsenic concentrations ( $\left.\mathrm{mg} \mathrm{L}^{-1}\right)$ of 20 rice genotypes

\begin{tabular}{|c|c|c|c|c|}
\hline \multirow{2}{*}{ Genotypes } & \multicolumn{3}{|c|}{ Arsenic concentration $\left(\mathrm{mg} \mathrm{L}^{-1}\right)$} & \multirow{2}{*}{ Genotypic means } \\
\hline & 0.75 & 1.0 & 1.25 & \\
\hline IR44595 & 68.92 ef & $80.76 \mathrm{bcd}$ & $84.30 \mathrm{~b}$ & 77.99 \\
\hline IR9209-26-2 & $73.15 \mathrm{cdf}$ & $81.59 \mathrm{~b}$ & $83.49 \mathrm{~b}$ & 79.81 \\
\hline Tie $90-1$ & $64.13 \mathrm{gh}$ & $70.87 \mathrm{i}$ & $72.41 \mathrm{hi}$ & 69.06 \\
\hline Aijiaonante 100 & $56.01 \mathrm{i}$ & $70.99 \mathrm{i}$ & $74.35 \mathrm{~g}$ & 67.14 \\
\hline Cocodire & $74.59 \mathrm{bcd}$ & 80.98 bc & $84.21 \mathrm{~b}$ & 79.86 \\
\hline Priscilla & $71.59 \mathrm{def}$ & $79.39 \mathrm{cdef}$ & 81.00 cde & 77.34 \\
\hline Jing 185-7 & $76.31 \mathrm{bc}$ & $78.97 \mathrm{def}$ & $81.49 \mathrm{~cd}$ & 78.97 \\
\hline Jinnvo No. 6 & 72.45 cde & $76.23 \mathrm{~g}$ & $79.70 \mathrm{de}$ & 76.13 \\
\hline BRRI dhan28 & $56.61 \mathrm{i}$ & $68.21 \mathrm{j}$ & $70.81 \mathrm{ij}$ & 65.22 \\
\hline BRRI dhan29 & $75.96 \mathrm{bc}$ & $77.43 \mathrm{fg}$ & $79.78 \mathrm{de}$ & 77.72 \\
\hline BRRI dhan 45 & $67.93 \mathrm{fg}$ & 79.53 cde & $80.63 \mathrm{de}$ & 76.00 \\
\hline BRRI dhan 47 & $55.80 \mathrm{i}$ & 59.771 & $61.35 \mathrm{k}$ & 58.89 \\
\hline HUA565 & $75.65 \mathrm{bcd}$ & $78.06 \mathrm{efg}$ & $79.51 \mathrm{e}$ & 77.77 \\
\hline HUA564 & $63.18 \mathrm{~h}$ & $64.50 \mathrm{k}$ & $69.07 \mathrm{j}$ & 65.62 \\
\hline Weed tolerant Rice & $76.72 \mathrm{bcd}$ & $80.28 \mathrm{bcd}$ & 81.22 cde & 79.42 \\
\hline BRRI dhan50 & $82.85 \mathrm{a}$ & $87.56 \mathrm{a}$ & $88.86 \mathrm{a}$ & 86.45 \\
\hline BRRI dhan61 & $74.55 \mathrm{bcd}$ & $87.05 \mathrm{a}$ & $88.90 \mathrm{a}$ & 83.44 \\
\hline BRRI dhan 49 & $78.04 \mathrm{~b}$ & - & - & 78.04 \\
\hline Jefferson & $75.70 \mathrm{bcd}$ & $81.15 \mathrm{bc}$ & $82.72 \mathrm{bc}$ & 79.89 \\
\hline BRRI dhan54 & - & $74.13 \mathrm{~h}$ & $77.37 \mathrm{f}$ & 75.71 \\
\hline Concentration means & 70.82 & 76.38 & 78.75 & \\
\hline LSD at 0.05 & & 0.68 & & 1.65 \\
\hline Standard deviation & 7.83 & 7.11 & 6.81 & \\
\hline Range & $55.80-82.85$ & $59.77-87.56$ & $61.35-88.90$ & \\
\hline
\end{tabular}

Within column different letters indicate significance differences among rice varieties (LSD test: $\mathrm{P}<0.05$ )

\subsection{Root-Shoot Biomass Reduction}

Genotypic mean of root-shoot biomass reduction was observed minimum (29.62\%) in BRRI dhan 47 followed by BRRI dhan45 (33.33\%), BRRI dhan54 (35.22\%) and BRRI dhan29 (37.37\%) (Table 6). Concentration means revealed that root-shoot biomass reduction was the minimum at 0.25 $\mathrm{mg} \mathrm{L}{ }^{-1}$ and the maximum at $1.50 \mathrm{mg} \mathrm{L}^{-1}$ although all concentration means are statistically dissimilar from each other except $0.75 \mathrm{mg} \mathrm{L}^{-1}$ to $1.0 \mathrm{mg} \mathrm{L}^{-1}$ and at $1.0 \mathrm{mg} \mathrm{L}^{-1}$ to at $1.25 \mathrm{mg} \mathrm{L}^{-1}$. Arsenic concentration level from $0.75 \mathrm{mg} \mathrm{L}^{-1}$ to $1.50 \mathrm{mg} \mathrm{\textrm {L } ^ { - 1 }}$ revealed the highest statistical differentiation among genotypes except $1.25 \mathrm{mg} \mathrm{L}^{-1}$. The highest range of root-shoot biomass reduction among the genotypes was observed at the concentration of $0.75 \mathrm{mg}$ $\mathrm{L}^{-1}$ followed by $1.0 \mathrm{mg} \mathrm{L}^{-1}, 0.5 \mathrm{mg} \mathrm{L}^{-1}, 1.25 \mathrm{mg} \mathrm{L}^{-1}$ and 0.25 $m g \mathrm{~L}^{-1}$ where as, standard deviation was the highest at the concentration of $0.75 \mathrm{mg} \mathrm{L}^{-1}$ followed by $0.5 \mathrm{mg} \mathrm{L}^{-1}, 1.25$ $\mathrm{mg} \mathrm{L}^{-1}, 1.0 \mathrm{mg} \mathrm{L}^{-1}$ and $0.25 \mathrm{mg} \mathrm{L}^{-1}$.

Root-shoot biomass was comparatively less affected at different doses of Arsenic in dose validation experiment. The minimum genotypic mean of root shoot-biomass reduction was observed in BRRI dhan47 (28.79\%) compared to IR9209-26-2 (47.41\%) and Jing 185-7 $(46.39 \%)$ (Table 7). The maximum root-shoot biomass reduction was observed at higher concentration $(1.25 \mathrm{mg}$ $\mathrm{L}^{-1}$ ) of arsenic in culture solution. The difference between tolerant variety (BRRI dhan47) and sensitive varieties (BRRI dhan45, and BRRI dhan29) for root-shoot biomass reduction was higher at $1.25 \mathrm{mg} \mathrm{L}^{-1}$ Arsenic. The range (31.60-52.76) and standard deviation (6.63) were also recorded high at $1.25 \mathrm{mg} \mathrm{L}^{-1}$ Arsenic. 
Table 6. Mean of root-shoot biomass reduction (\%) over different concentration $\left(\mathrm{mg} \mathrm{L}^{-1}\right)$ of arsenic in dose response experiment

\begin{tabular}{|c|c|c|c|c|c|c|c|}
\hline \multirow{2}{*}{ Genotypes } & \multicolumn{6}{|c|}{ Arsenic concentration $\left(\mathrm{mg} \mathrm{L}^{-1}\right)$} & \multirow{2}{*}{ Genotypic means } \\
\hline & 0.25 & 0.50 & 0.75 & 1.0 & 1.25 & 1.50 & \\
\hline BRRI dhan29 & $10.0 \mathrm{a}$ & $30.5 \mathrm{a}$ & $43.2 \mathrm{a}$ & $43.9 \mathrm{a}$ & $45.3 \mathrm{a}$ & $51.3 \mathrm{a}$ & 37.37 \\
\hline BRRI dhan45 & $9.6 \mathrm{a}$ & $24.9 \mathrm{a}$ & $37.5 \mathrm{ab}$ & $38.3 \mathrm{ab}$ & $40.8 \mathrm{a}$ & $48.8 \mathrm{ab}$ & 33.33 \\
\hline BRRI dhan47 & $15.5 \mathrm{a}$ & $20.9 \mathrm{a}$ & $30.4 \mathrm{~b}$ & $33.3 \mathrm{~b}$ & $34.6 \mathrm{~b}$ & $43.1 \mathrm{c}$ & 29.62 \\
\hline BRRI dhan54 & $14.9 \mathrm{a}$ & $30.4 \mathrm{a}$ & $37.8 \mathrm{ab}$ & $38.6 \mathrm{ab}$ & $42.4 \mathrm{a}$ & $47.3 \mathrm{~b}$ & 35.22 \\
\hline Concentration means & 12.49 & 26.67 & 37.23 & 38.52 & 40.77 & 47.63 & \\
\hline $\operatorname{LSD}(0.05)$ & \multicolumn{6}{|c|}{3.28} & 2.67 \\
\hline Standard Deviation & 3.10 & 4.65 & 5.24 & 4.34 & 4.53 & 3.45 & \\
\hline Range & $9.6-15.5$ & $20.9-30.5$ & $30.4-43.2$ & $33.3-43.9$ & $34.6-45.3$ & $43.1-51.3$ & \\
\hline
\end{tabular}

Within column different letters indicate significance differences among rice varieties (LSD test: $\mathrm{P}<0.05$ )

Table 7. Mean root-shoot biomass reduction (\%) over three levels of arsenic concentrations $\left(\mathrm{mg} \mathrm{L}^{-1}\right)$ of 20 rice genotypes

\begin{tabular}{|c|c|c|c|c|}
\hline \multirow{2}{*}{ Genotypes } & \multicolumn{3}{|c|}{ Arsenic concentration $\left(\mathrm{mg} \mathrm{L}^{-1}\right)$} & \multirow{2}{*}{ Genotypic Mean } \\
\cline { 2 - 4 } & 0.75 & 1.0 & 1.25 & 29.13 \\
\hline IR44595 & $25.98 \mathrm{fg}$ & $28.65 \mathrm{l}$ & $32.73 \mathrm{mn}$ & 47.41 \\
\hline IR9209-26-2 & $43.24 \mathrm{a}$ & $46.28 \mathrm{ab}$ & $52.75 \mathrm{a}$ & 33.72 \\
\hline Tie 90-1 & $28.47 \mathrm{efg}$ & $34.33 \mathrm{hij}$ & $38.34 \mathrm{jkl}$ & 36.42 \\
\hline Aijiaonante 100 & $29.83 \mathrm{defg}$ & $36.79 \mathrm{ghi}$ & $42.66 \mathrm{fgh}$ & 44.08 \\
\hline Cocodire & $42.07 \mathrm{a}$ & $43.60 \mathrm{bcde}$ & $47.04 \mathrm{cde}$ & 39.23 \\
\hline Priscilla & $35.40 \mathrm{bcd}$ & $38.92 \mathrm{fg}$ & $42.35 \mathrm{ghi}$ & 46.39 \\
\hline Jing 185-7 & $41.52 \mathrm{a}$ & $47.20 \mathrm{a}$ & $50.39 \mathrm{abc}$ & 36.38 \\
\hline Jinnvo No. 6 & $34.66 \mathrm{~cd}$ & $36.90 \mathrm{gh}$ & $38.97 \mathrm{ijk}$ & 35.02 \\
\hline BRRI dhan28 & $31.58 \mathrm{def}$ & $33.33 \mathrm{jk}$ & $40.14 \mathrm{hij}$ & 42.06 \\
\hline BRRI dhan29 & $31.34 \mathrm{def}$ & $45.59 \mathrm{abc}$ & $49.23 \mathrm{bcd}$ & 42.15 \\
\hline BRRI dhan45 & $31.90 \mathrm{de}$ & $44.87 \mathrm{abcd}$ & $49.69 \mathrm{abc}$ & 28.79 \\
\hline BRRI dhan47 & $24.94 \mathrm{~g}$ & 29.861 & $31.61 \mathrm{n}$ & 33.85 \\
\hline HUA565 & $32.31 \mathrm{de}$ & $33.54 \mathrm{jjk}$ & $35.74 \mathrm{klm}$ & 33.56 \\
\hline HUA564 & $30.54 \mathrm{defg}$ & $33.34 \mathrm{jk}$ & $36.84 \mathrm{jkl}$ & 42.34 \\
\hline Weed tolerant Rice & $39.04 \mathrm{abc}$ & $42.08 \mathrm{def}$ & $45.82 \mathrm{def}$ & 30.69 \\
\hline BRRI dhan50 & $26.48 \mathrm{efg}$ & $30.46 \mathrm{kl}$ & $35.32 \mathrm{~lm}$ & 41.25 \\
\hline BRRI dhan61 & $27.51 \mathrm{efg}$ & $44.00 \mathrm{abcde}$ & $52.29 \mathrm{ab}$ & 41.12 \\
\hline BRRI dhan49 & $41.11 \mathrm{ab}$ & - & - & \\
\hline Jefferson & $31.05 \mathrm{def}$ & $40.79 \mathrm{ef}$ & $44.28 \mathrm{efg}$ & 38.73 \\
\hline BRRI dhan54 & - & $42.59 \mathrm{cde}$ & $47.34 \mathrm{cde}$ & 44.99 \\
\hline Concentration means & 33.20 & 39.02 & 43.14 & \\
\hline LSD at 0.05 & & 1.09 & & \\
\hline Standard deviation & 5.66 & 6.18 & 6.63 & \\
\hline Range & $24.94-43.24$ & $28.66-47.21$ & $31.60-52.76$ & \\
\hline
\end{tabular}

Within column different letters indicate significance differences among rice varieties (LSD test: $\mathrm{P}<0.05$ )

\section{Discussion}

Arsenic is generally phyto-toxic and negatively affects plant growth [1], particularly yield and biomass production in rice are greatly reduced at elevated Arsenic concentrations, often leading to death of plant population [8-10].Irrigation with Arsenic contaminated ground water greatly affects early growth stages of rice plants inhibiting phosphorus uptake from the soil. However, arsenate is an analog of inorganic phosphate (Pi) and is easily transported across the plasma lemma by Pi transporter (PHT) protein [28-29]. When phosphate competes with arsenate for uptake, arsenate toxicity is lower under high phosphate condition. Phosphate free hydroponic solutions [30] therefore, provide the clearest insights into the potency of externally supplied Arsenic on whole plant growth because arsenate is out compete phosphate for entry into the plant [31].

During early growth stage root and shoot development, particularly, root length, shoot length and root-shoot biomass reduced significantly at elevated arsenic concentration in phosphate free culture solution compared to control. Arsenic stress reduced the phenotypic expression of all the seedling traits; shoot length, root length and root-shoot biomass (Figure 1). Although, the effect of arsenic was more prominent on root length compared to the shoot length and root-shoot biomass indicating lesser severity of arsenic on shoot growth and biomass. Abedin et al. [32] observed that plant roots were the first tissue of contact to Arsenic in nutrient media, where the metalloid inhibited root extension and proliferation. Similar adverse effect of arsenic on root length was observed by Carbonell-Barrachina et al. [33]. 

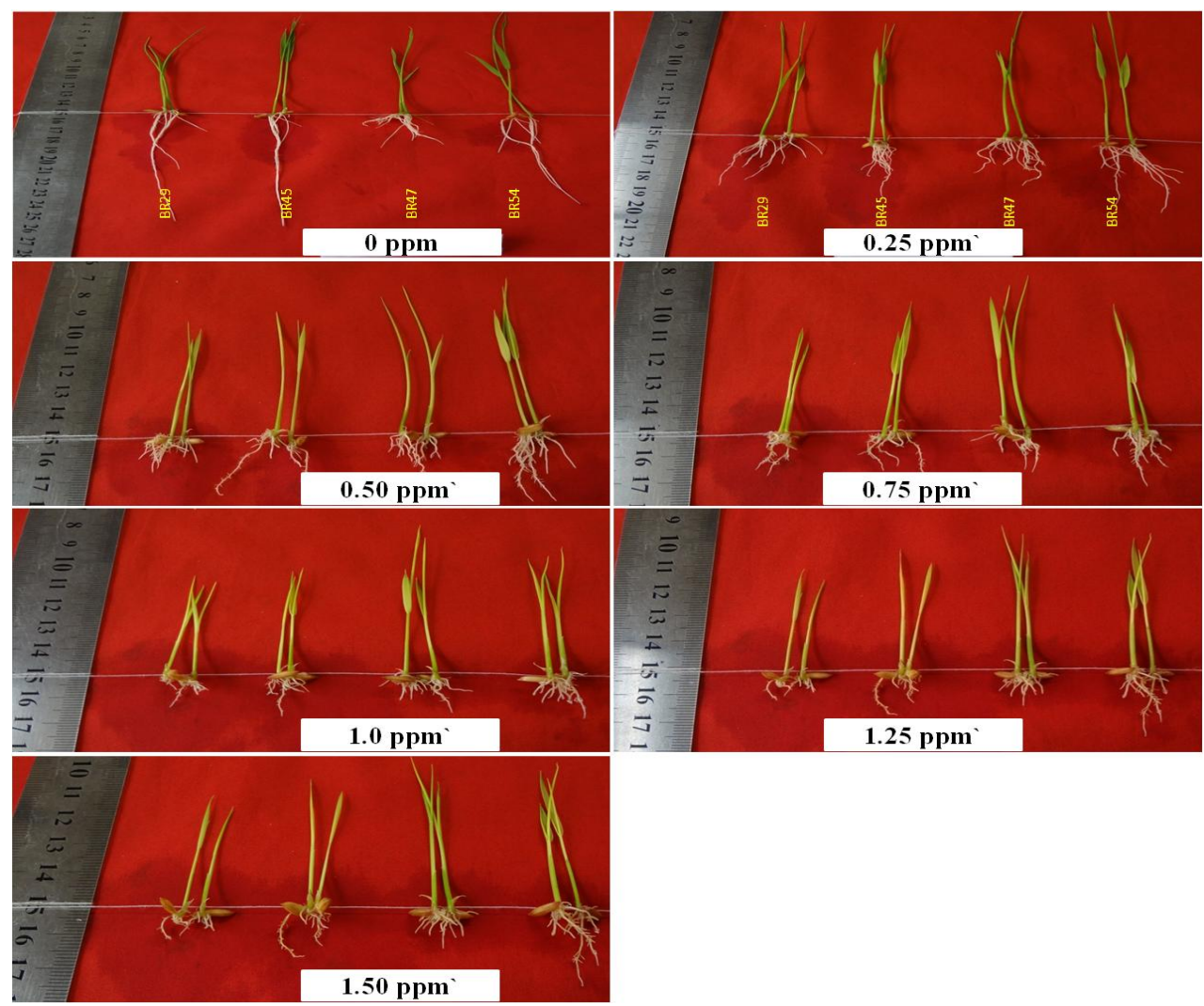

Figure 1. Images of seedling showing effect of different levels of arsenic concentration on early growth stage of different rice genotypes (BR29=BRRI dhan29, BR45=BRRI dhan45, BR47=BRRI dhan47 and BR54=BRRI dhan54, respectively, from left to right in the photographs)

The dose response experiment with six level of Arsenic starting from $0.25 \mathrm{mg} \mathrm{L}^{-1}$ to $1.5 \mathrm{mg} \mathrm{L}^{-1}$ over two tolerant varieties (BRRI dhan47 and BRRI dhan54) and two susceptible varieties (BRRI dhan29 and BRRI dhan45) showed maximum statistical differentiation, range and standard deviation at $0.75-1.25 \mathrm{mg} \mathrm{L}^{-1}$ arsenic treatment for shoot length reduction, root-shoot biomass reduction but for root length reduction arsenic concentrations starting from $0.50 \mathrm{mg} \mathrm{L}^{-1}$ to $1.25 \mathrm{mg} \mathrm{L}^{-1}$ Arsenic were found highly responsive to differentiate genotypes. Considering mean reduction, standard deviation and range of the genotypes in all three growth traits, the $0.75-1.25 \mathrm{mg} \mathrm{L}^{-1}$ Arsenic concentrations were found suitable to screen for arsenic tolerance in rice at early growth stage. Also the tolerant variety, BRRI dhan 47 showed minimum reduction in all three growth traits at $0.75-1.25 \mathrm{mg} \mathrm{L}^{-1}$ Arsenic level.

The dose validation experiment with $0.75 \mathrm{mg} \mathrm{L}^{-1}, 1.0$ $\mathrm{mg} \mathrm{L}^{-1}$ and $1.25 \mathrm{mg} \mathrm{L}^{-1}$ over a set of 20 diverse genotypes including BRRI dhan 47 as tolerant check and BRRI dhan 29 and BRRI dhan 45 as susceptible checks also showed minimum and maximum reduction, respectively in all three growth traits. The reduction was the most acute, highly significant and maximum segregation among the genotypes was found at $1.25 \mathrm{mg} \mathrm{L}^{-1}$ Arsenic in both shoot length reduction and root-shoot biomass reduction traits. Similar trend of response for root length reduction was observed at $1.0 \mathrm{mg} \mathrm{L}^{-1}$ Arsenic concentration. Reduced root length at early growth stage under arsenic stress in the present study was parallel to the findings of Dasgupta et al. [20] who identified $13.3 \mu \mathrm{M}$ (i.e $1.0 \mathrm{mg} \mathrm{L}^{-1}$ ) arsenate was suitable dose to screen root length reduction trait of diverse genotypes under hydroponic arsenic stress condition. Abedin et al. [32] reported significant reduction in root biomass at $>1 \mathrm{mg} \mathrm{L}^{-1}$ Arsenic(V).Marin et al. [34] found significant reduction of rice shoot length when arsenite or monomethyl arsenic acid was applied at a dose of $0.8 \mathrm{mg}$ $\mathrm{L}^{-1}$. Therefore, this study suggested that $1.25 \mathrm{mg} \mathrm{L}^{-1}$ of Arsenic could be effective and efficient for discriminating tolerant and sensitive rice genotypes considering all three growth traits (root length, shoot length and root-shoot biomass)against arsenic phytotoxicity at early growth stage in hydroponic culture.

\section{Conclusions}

Arsenic (arsenate compound) was found to be toxic to seedling growth of rice. Root length was significantly reduced with increasing arsenic concentrations in the hydroponic solution. Effective and efficient differentiation was observed at $1.25 \mathrm{mg} \mathrm{L}^{-1}$ Arsenic for shoot length and root-shoot biomass, while $1.0 \mathrm{mg} \mathrm{L}^{-1}$ Arsenic was sufficient for root length trait. Taking into account of the above discussions, it could be concluded that the concentration of $1.25 \mathrm{mg} \mathrm{L}^{-1}$ of Arsenic was the best for detection of differences between tolerant and sensitive rice genotypes 
at early growth stage considering all three growth traits (root length, shoot length and root-shoot biomass) against arsenic toxicity which would be a simple and quick screening protocol. Hence, this study would provide a good scope for identifying genotypes with higher level of tolerance to Arsenic stress from mass screening of segregating or diverse sets of genotypes. This study also further proved BRRI dhan47's promise to be used as a potential donor in the future rice breeding programmes for arsenic phyto-toxicity tolerance.

\section{Acknowledgements}

We acknowledge Food for Progress Project (RCC-388-2009) of BSMRAU - Cornel University for financial support of this study. We also acknowledge BRRI authorities for providing workspace and materials used for this study.

\section{REFERENCES}

[1] Kabata-Pendias, A. and Pendias, H. 1991. Trace elements in soils and plants. CRC Press, Boca Raton, Florida.

[2] Dat, J., Vandenabeele, S., Vranova, E., Montagu, M.V., Inze, D., Breusegem, F.V. 2000. Dual action of the active oxygen species during plant stress responses. Cell Mol Life Sci. 57: 779-795.

[3] Stoeva, N., Berova, M., Zlatev, Z. 2003. Physiological response of maize (Zea Mays L.) to different levels of as contamination. Biol Plant. 47: 449-452.

[4] Abbas, G., Murtaza, B., Bibi, I., Shahid, M., Niazi, N. K., Khan, M. I., Amjad, M., Hussain, M., Natasha. 2018. Arsenic Uptake, Toxicity, Detoxification, and Speciation in Plants: Physiological, Biochemical, and Molecular Aspects. Int. J. Environ. Res. Public Health. 15: 59.

[5] Panaullah, G. M., Alam, T., Hossain, M. B., Loeppert, R. H., Lauren, J. G., Meisner, C. A., Ahmed, Z. U., Duxbury, J. M. 2009. Arsenic toxicity to rice (Oryza sativa L.) in Bangladesh. Plant Soil. 317: 31-39.

[6] Rahman, M. A., Hasegawa, H., Rahman, M. M., Miah, M. A. M., Tasmin, A. 2008. Straighthead disease of rice (Oryza sativa L.) induced by arsenic toxicity. Environ Exp Bot. 62: 54-59.

[7] Syed, M. A., Iftekharuddaula, K. M., Mian, M. A. K., Rasul, M. G., Rahmam, G. K. M. M, Panaullah, G. M., Lauren, J. G., Duxbury, J. M., Biswas, P. S. 2016. Main effect QTLs associated with arsenic phyto-toxicity tolerance at seedling stage in rice (Oryza sativa L.); Euphytica, 209: 805-814.

[8] Bakhat, H. F., Zia, Z., Fahad, S., Abbas, S., Hammad, H. M., Shahzad, A. N., Abbas, F., Alharby, H., Shahid, M. 2017. Arsenic uptake, accumulation and toxicity in rice plants: Possible remedies for its detoxification: A review. Environ. Sci. Pollut. Res. 24: 9142-9158.
[9] Rahman, M. A., Hasegawa, H., Rahman, M. M., Islam, M. N., Miah, M. A. M. 2007. Effect of arsenic on photosynthesis, growth and yield of five widely cultivated rice (Oryza sativa L.) varieties in Bangladesh. Chemosphere. 67: 1072-1079.

[10] Akter, K. F., Owens, G., Davey, D. E., Naidu, R. 2005. Arsenic speciation and toxicity in biological systems. Reviews Environ. ContaminToxico. 184: 97-149.

[11] Vromman, D., Lutts, S., Lefèvre, I., Somer, L., De Vreese, O., Šlejkovec, Z., Quinet, M. 2013. Effects of simultaneous arsenic and iron toxicities on rice (Oryza sativa L.) development, yield-related parameters and as and $\mathrm{Fe}$ accumulation in relation to as speciation in the grains. Plant Soil 371: 199-217.

[12] Nath, S., Panda, P., Mishra, S., Dey, M., Choudhury, S., Sahoo, L., Panda, S. K. 2014. Arsenic stress in rice: Redox consequences and regulation by iron. Plant Physiol. Biochem. 80: 203-210.

[13] Chen, Y., Ahsan, H. 2004. Cancer burden from arsenic in drinking water in Bangladesh. Am J Public Health. 94: 741-744.

[14] Gupta, D. K., Tiwari, S., Razafindrabe, B. H. N., Chatterjee, S. 2017. Arsenic contamination from historical aspects till present situation. In Arsenic Contamination in the Environment: The Issues and Solutions; Gupta, D. K., Chatterjee, S., Eds.; Springer International Publishing AG: Cham, Switzerland, pp. 1-12.

[15] Rout, G. R., Das, P. 2002. Rapid hydroponic screening for molybdenum tolerance in rice through morphological and biochemical analysis. Rostl Vyroba. 48: 505-512.

[16] Liu, W. J., McGrath, S. P., Zhao, F. J. 2014. Silicon has opposite effects on the accumulation of inorganic and methylated arsenic species in rice. Plant Soil 376: 423-431.

[17] Devine, T. E. 1982. Genetic fitting of crops to problem soil. In: Christiansen N, Lewis CF. (eds): Breeding plants in less favourable environments. Wiley, New York. p. 143-173.

[18] Farooq, M. A., Islam, F., Ali, B., Najeeb, U., Mao, B., Gill, R. A., Yan, G., Siddique, K. H. M., Zhou, W. 2016. Arsenic toxicity in plants: Cellular and molecular mechanisms of its transport and metabolism. Environ. Exp. Bot. 132: 42-52.

[19] Carver, B. F., Inskeep, W. P., Wilson, N. P., Westerman, R. L. 1998. Seedlings' tolerance to aluminium toxicity in hard red winter wheat germplasm. Crop Sci. 28: 463-467.

[20] Dasgupta, T., Hossain, S. A., Meharg, A. A., Price, A. H. 2004. An arsenate tolerance gene on chromosome 6 of rice. New Phytol. 163: 45-49.

[21] Bastías, J. M., Beldarrain, T. 2016. Arsenic translocation in rice cultivation and its implication for human health. Chil. J. Agric. Res. 76: 114-122.

[22] Pinson, S. R. M., Tarpley, L., Yan, W., Yeater, K., Lahner, B., Yakubova, E., Huang, X. Y., Zhang, M., Guerinot, M. L., Salt, D. E. 2015. Worldwide genetic diversity for mineral element concentrations in rice grain. Crop Sci. 55: 294-311.

[23] Meharg, A. A. 2005. Venomous Earth: How arsenic caused the world's worst mass poisoning: Macmillan Houndmills, England. 
[24] Shri, M., Singh, P. K., Kidwai, M., Gautam, N., Dubey, S., Verma, G., Chakrabarty, D. 2019. Recent advances in arsenic metabolism in plants: current status, challenges and highlighted biotechnological intervention to reduce grain arsenic in rice. Metallomics 20: 519-532.

[25] Deng, F., Yu, M., Martinoia, E., Song, W. Y. 2019. Ideal Cereals with Lower Arsenic and Cadmium by Accurately Enhancing Vacuolar Sequestration Capacity. Front Genet. 10: 322 .

[26] Biswas, P. S., Anisuzzaman, A., Syed, M. A., Islam, A., Kabir, M. S., Panaulla, G. M., Laurence, J., and Duxbury, J. M. 2014. BRRI dhan47: A potential donor for arsenic phytotoxicity tolerance in rice. Bangladesh Rice Research Abstract. p. 28. Bangladesh Rice Research Institute, Gazipur, Bangladesh.

[27] Foy, C. D., Chaney, R. L., White, M. C. 1978. The physiology of metal toxicity in plants. Ann Rev Plant Physiol. 29: 511-566.

[28] Mitra1, A., Chatterjee, S., Moogouei, R., Gupta, D. K. 2017. Arsenic Accumulation in Rice and Probable Mitigation Approaches: A Review. Agronomy 7: 67.

[29] Barcelo, J., Poschenrieder, Ch. 1990. Plant-water relations as affected by heavy metal stress: A review. J Plant Nutr. 13: $1-37$.

[30] Kalita, J., Pradhan, A. K., Shandilya, Z. M., Tanti, B. 2018. Arsenic Stress Responses and Tolerance in Rice: Physiological, Cellular and Molecular Approaches; Rice Science 25: 235-249.

[31] Niazi, N. K., Bibi, I., Shahid, M., Ok, Y. S., Burton, E. D., Wang, H., Shaheen, S. M., Rinklebe, J., Lüttge, A. 2018. Arsenic removal by perilla leaf biochar in aqueous solutions and groundwater: An integrated spectroscopic and microscopic examination. Environ. Pollut. 232: 31-41.

[32] Abedin, M. D. J., Cresser, M. S., Meharg, A. A., Feldmann, J., Cotter-Howells, J. 2002. Arsenic accumulation and metabolism in rice (Oryza sativa L.). Environ Sci Techno. 36: $962-968$.

[33] Carbonell-Barrachina, A. A., Burlo, F., Burgos-Hernandez, A., Lopez, E., Mataix, J. 1997. The influence of arsenite concentration on arsenic accumulation in tomato and bean plants. SciHortic. 71: 167-176.

[34] Marin, A. R., Masscheleya, P. H., Parrick, W. H. Jr. 1992. The influence of chemicals form and concentration of arsenic on rice growth and tissue arsenic concentration. Plant Soil 139: 175-183. 